\title{
Retour des expériences individuelles de stage pour un apprentissage collaboratif
}

\author{
JACQUES GRANDJEAN \\ Maître de conférences, doyen des programmes de Mastères Spécialisés, \\ École nationale des ponts et chaussées (École des Ponts ParisTech) \\ jacques.grandjean@enpc.fr
}

\section{Résumé}

S'appuyant sur des théories du groupe et des apports théorico-pratiques sur la méthode de conduite de groupe, l'auteur montre en quoi l'expérience du groupe et du travail de groupe, régulée par un conducteur de groupe expérimenté, contribue à l'apprentissage de la complexité et de co-apprentissage permettant l'acquisition de compétences psychosociales et d'aptitudes au travail collaboratif.

Il s'appuie sur l'analyse d'un séminaire de retour d'expérience du stage d'ingénieur pour montrer qu'en mettant le groupe, lieu de confrontation des différences, au centre du dispositif, il est possible de développer les capacités des étudiants au co-apprentissage et d'accompagner l'individualisation de leurs dynamiques professionnelles.

\section{Summary}

Based on the group's theories and theoretical-practical contributions to the method of group leadership, the author shows how the group's experience and group work is a learning factor of complexity and co-learning that enables the acquisition of psychosocial skills and collaborative skills.

He uses the analysis of a feedback seminar of the engineer's internship to show that by putting the group, the place of confrontation of differences, at the center of the device it is possible to develop the students' abilities to co-learn and to accompany the individualization of their professional dynamics.

Mots-clés

Retour d'expérience, diversité, groupe, co-apprentissage, réflexivité

Key words

Experience feed-back, diversity, co-learning, reflexivity 


\section{Introduction}

L'internationalisation, l'intégration systémique des processus et la numérisation des activités transforment les organisations et les métiers. C'est ainsi que la transformation des métiers de la construction par la transition énergétique, la transformation des systèmes de mobilité ou l'intégration des systèmes urbains a conduit l'École des Ponts ParisTech à créer des formations spécialisées dans ces domaines pour répondre aux besoins des entreprises. Pour s'adapter à ce nouvel environnement, l'ingénieur doit développer des compétences collaboratives au sein de systèmes professionnels complexes intégrant différents métiers, des structures d'organisation multiples et des cultures hétérogènes.

Dans son livre blanc «apprendre à manager le travail », l'Agence Nationale d'Amélioration des Conditions de Travail (ANACT) (2017) souligne l'insuffisance des écoles d'ingénieurs et de management à « développer la capacité des ingénieurs à s'intégrer dans une organisation, à l'animer et à la faire évoluer » relativement peu initiés dans les écoles à une réflexion et à un outillage qui leur permettent la prise en compte de la «complexité humaine et organisationnelle » dans leur future activité. Dans ces écoles, «les programmes relatifs à la compréhension de la dimension humaine et collective des organisations ainsi que la conduite des changements et des équipes représentent une très faible part des cursus » (ANACT, 2017).

Dès lors, l'exploitation des stages et missions exercés en milieu professionnel (la moitié de la scolarité à l'école des Ponts ParisTech) constitue un enjeu pédagogique important pour développer des compétences psychosociales nécessaires pour faire face à la complexité humaine et organisationnelle. Cette complexité repose notamment sur la diversité qui, par exemple, au regard des stages effectués par les étudiants ingénieurs de l’École, se décline en termes de diversité culturelle liée aux origines sociales et géographiques ou relative aux métiers exercés (valeurs, représentations métier, hiérarchie, travail en groupe, etc.) ; diversité des fonctions exercées et diversité des structures d'organisation et des contextes socio-économiques des pays dans lesquels s'exercent les activités. Les effets résultant de cette diversité, une des composantes de la complexité, peuvent être source de satisfaction et de stimulation, mais aussi, parfois causes de mal-être, d'isolement et d'échec. Il y a donc nécessité de contribuer à la prévention des risques psychosociaux pour les jeunes professionnels, comme des situations d'épuisement professionnel, de conflits relationnels ou d'abus de pouvoir. L'objet de cet article 
est de vérifier en quoi, un retour d'expérience en groupe, contribue à l'acquisition de savoirs permettant d'analyser des situations professionnelles complexes et d'interagir dans des équipes d'une grande diversité culturelle, statutaire et des fonctions.

A cette fin, dans le cadre d'une démarche d'action-recherche ${ }^{1}$, nous analyserons a posteriori le dispositif intitulé « Séminaire de retour d'expérience du stage d'ingénieur » présenté dans une première partie. Au préalable, nous présenterons les théories du groupe et du travail de groupe qui constituent le cadre théorique soutenant la mise en place du dispositif et nous préciserons les questions issues de ces éléments théoriques. Ensuite, nous utiliserons les données issues des questionnaires d'évaluation auxquels ont répondu 183 étudiants ingénieurs, pour évaluer la capacité de ce dispositif de formation à contribuer au développement de compétences psychosociales, en particulier celles concernant le travail en groupe. Les données utilisées sont issues de questionnaires d'évaluation réalisés dans un but d'amélioration continue du dispositif et non dans le cadre d'une recherche académique ; cela pose des limites aux résultats de cette réflexion que nous discuterons.

\section{Le séminaire de retour d'expérience du stage ingénieur}

La formation des ingénieurs de l'École des Ponts ParisTech comporte un stage entre la deuxième et la troisième année. Ce stage dure deux mois ou une année pour $90 \%$ des étudiants. Le séminaire de retour d'expérience est situé après la fin du stage et en début de troisième année. Il constitue un sas entre l'expérience professionnelle et le retour à la vie étudiante.

Près de 120 étudiants sur les 250 de la promotion d'ingénieurs participent à ce séminaire obligatoire pour ceux qui réalisent leur troisième année à l'école. Ils sont issus des six départements de spécialité ${ }^{2}$ avec environ deux tiers d'étudiants étrangers en double-diplôme. Plus d'une vingtaine de nationalités sont représentées. Le but de ce séminaire est de se saisir de l'expérience de terrain des étudiants pour leur fournir une grille d'analyse des interactions entre individus, groupes et organisations et de contribuer à leur orientation professionnelle, en particulier dans le choix et la préparation de leur prochain stage de fin d'étude.

Les 3 principaux objectifs pédagogiques du séminaire sont :

\footnotetext{
${ }^{1}$ L'action-recherche pose le primat du travail clinique sur le terrain mais également la production de savoirs généralisables à d'autres contextes (Rouchy et Soula-Desroche, 2004).

${ }^{2}$ Départements de spécialité : Ville, environnement, transport ; Génie civil et construction ; Génie Industriel ; Génie Mécanique et Matériaux ; Informatique et ingénierie mathématique ; Économie, gestion, finance.
} 
1. améliorer la connaissance de soi dans un fonctionnement institutionnel (compétences relationnelles, rapport à l'autorité, fonctionnement en groupe, aptitude à défendre un point de vue, à négocier ou à gérer son temps, etc.) ;

2. acquérir une méthode d'analyse en groupe d'une situation professionnelle par la confrontation à l'expérience des pairs et par des apports théoriques notamment ceux de la psychosociologie ;

3. réfléchir à sa dynamique professionnelle et formaliser des critères de choix.

À ces objectifs s'ajoute l'intention, dans le cadre de la prévention des risques psycho-sociaux, d'offrir une expérience de groupe de soutien où peuvent se travailler en confiance des situations professionnelles difficiles.

Le dispositif dure trois jours et demi et comporte :

- Deux journées d'ateliers d'analyse de pratique en groupes restreints de 12 à 15 étudiants avec des analyses de situations professionnelles (relationnelles et managériales) vécues par les étudiants pendant leur stage et des apports théoriques du champ de la psychosociologie notamment sur les structures d'organisation, la dynamique de groupe et la conduite de réunion. Certains de ces apports sont sous la forme d'exposés enregistrés et disponibles sur le site de ressources éducatives de l'établissement ;

- une demi-journée de réflexion sur la dynamique personnelle et professionnelle en petits groupes ;

- une demi-journée d'approfondissements thématiques où les étudiants identifient, choisissent et approfondissent en groupe une douzaine de thèmes issus des situations analysées ;

- une demi-journée de restitution en plénière de ces groupes thématiques accompagnée d'un débat avec l'ensemble de la promotion.

La diversité des situations traitées dans les analyses de situations provient de contextes très variés : chantiers BTP, banques d'affaire, bureaux d'étude, laboratoires de recherche, agences d'architecture, entreprises internationales du luxe, start-up, associations humanitaires... Elle s'origine aussi dans la multiplicité des problématiques : intégration dans une équipe, positionnement dans une petite structure charismatique, relations hiérarchiques, conflits entre groupes socio-professionnels, compétition, multiculturalité, place des femmes, travail mal encadré, sous-emploi ou surexploitation, rémunération, gestion du temps, gestion du stress et de la fatigue, violence verbale, conflits de valeurs, place de la vie privée, etc. Les étudiants sont 
notés en fonction de leur participation dans les analyses de situation, des productions collectives de la plénière finale et d'une analyse d'une situation écrite et individuelle.

La dimension du groupe est abordée selon plusieurs modalités :

- les exposés concernant la dynamique et la conduite de groupe ;

- les analyses de situations mettant en évidence le fonctionnement des groupes et des équipes ainsi que les relations entre réunions et structures d'organisation ;

- l'expérience d'une conduite de groupe par des professionnels de l'animation ;

- les animations de certaines séquences que les étudiants assurent eux-mêmes ;

- des temps centrés sur la dynamique et le fonctionnement du groupe ici et maintenant ; ils visent à en réguler le fonctionnement si nécessaire et à éclairer le fonctionnement d'un groupe de travail.

Les intervenants, consultants ou enseignants-chercheurs, qui conduisent les ateliers ont en commun une formation et une pratique d'intervention psychosociologique, c'est-à-dire qu'ils interviennent auprès de professionnels et d'équipes dans des organisations au travers de divers dispositifs tels que l'analyse des pratiques, la régulation, l'accompagnement au changement, la consultation institutionnelle et la supervision d'équipes.

\section{Le cadre conceptuel}

Le cadre conceptuel se centrera sur quatre aspects de la dimension groupale des interactions en situation d'apprentissage :

- Le groupe comme lieu d'expérience de la diversité et des différences ;

- Le groupe support d'apprentissage et d'évolution ;

- La conduite de groupe comme régulation de la dynamique de groupe ;

- Le retour d'expérience comme soutien au processus de construction identitaire.

\subsection{Le groupe comme lieu d'expérience de la diversité et des différences}

Divers travaux ont mis en évidence que le groupe est un lieu où peuvent se penser les interactions et s'actualiser les conflits entre les dimensions personnelles (histoire, intérêts, culture, formation, valeurs, motivations, etc.), les dimensions du fonctionnement de l'équipe, les dimensions de l'organisation, ainsi que les valeurs, enjeux et logiques de l'institution qui traversent chaque individu dans l'exercice quotidien de son action (Kessar, 2015). C'est le lieu 
de construction de représentations collectives que chaque participant peut introjecter (Rouchy, 1998). Le groupe constitue le cadre par excellence pour travailler sur les représentations culturelles mais aussi les rapports inter et transculturels (Le Roy, 2017).

Le groupe est ainsi le lieu pour travailler les différences générées par la diversité des personnes, des origines, des cultures, des métiers et des appartenances des individus le constituant. Dans un groupe qui réunit des étudiants originaires du monde entier se pose d'emblée la question de la différence. Cette différence apparaît non seulement dans la diversité des références culturelles, mais aussi au regard d'une hiérarchisation explicite ou implicite entre les membres du groupe liée au sexe, à l'école ou à l'université d'origine, au pays d'origine et/ou aux classes sociales dont sont issus les étudiants. Les différences entre les membres du groupe sont potentiellement un objet de travail. Toutefois, on évite d'aborder directement ce thème. L'identité du sujet est fragilisée dans un groupe, et les différences mises en exergue augmentent cette fragilité. Nul individu n'est prêt d'emblée à exposer ce qui l'affaiblit.

La confrontation des différences provient des situations professionnelles rapportées dans le lieu d'analyse. Il s'agit de situations où les étudiants ont été aux prises avec des conflits générés par les différences de statut social ou professionnel, de logiques métiers, de modèles organisationnels ou de modèles relationnels, par exemple :

- un Français ayant intériorisé le modèle hiérarchique bureaucratique est déstabilisé par un modèle de fonctionnement américain moins hiérarchisé,

- un étudiant ne comprend pas que sa compétence soit insuffisante pour lui donner autorité dans une structure très hiérarchisée fondée sur le rang ou le statut,

- un autre ne trouve pas sa place dans une équipe multiculturelle, etc.

La diversité n'est pas seulement externe à l'individu. Elle est aussi intrapsychique : une personne peut se sentir « déchirée » entre des cadres de référence contradictoires issus de ses appartenances à différents groupes. Par exemple, un conflit de loyauté peut intervenir lors d'un choix nécessaire entre les valeurs de la famille d'origine et celles du nouveau groupe socioprofessionnel dans lequel l'individu s'intègre. Ce conflit pourra se manifester dans l'orientation professionnelle (De Gaulejac, 1992).

\subsection{Le groupe support d'apprentissage et d'évolution}

Le groupe est un lieu d'apprentissage selon certaines conditions. La participation à un groupe ne suffit pas pour qu'un individu se décentre de lui-même, pour qu'il accepte les points de vue des autres et qu'il remette en question ses représentations de lui-même et du monde. Il faut, 
d'une part, l'amorce d'un processus collectif de tissage des relations intersubjectives et, d'autre part, une tension entre une homogénéité facilitant les échanges et une hétérogénéité génératrice d'une conflictualité féconde (Meirieu, 1997).

La diversité des interprétations et la confrontation de points de vue variés contribuent à approfondir les analyses et à mieux comprendre les différences de comportement des acteurs de la situation rapportée. Elle est aussi une occasion de prendre conscience des différences au sein même du groupe, différences qui peuvent alors être dites et questionnées pour éviter qu'elles soient un frein aux échanges.

À travers l'analyse d'une situation, les différences peuvent apparaître comme un enrichissement formatif et non plus comme facteur conflictuel ou fragilisant si la conduite du groupe est suffisamment contenante.

\subsection{La conduite de groupe comme régulation de la dynamique de groupe}

La conduite d'un groupe nécessite d'être attentif à la dynamique de groupe pour en faire un ressort d'apprentissage. Or, cela suppose une compréhension minimum des processus à l'œuvre dans la situation groupale. Didier Anzieu (1999) note que la réalité d'un groupe s'effectue à deux niveaux : celui de la tâche commune et celui des émotions communes. Le premier niveau est conscient, rationnel centré autour des objectifs et de la tâche communs. Par exemple, dans le cadre d'un projet, le groupe agit pour produire les résultats attendus. Le second niveau souvent inconscient concerne la vie émotionnelle et affective. C'est cette dimension qui fait la complexité et la richesse des phénomènes de groupe. Le simple fait d'être intégré dans un groupe produit des comportements qui, s'ils sont compris et bien régulés, renforcent la vie du groupe et sa créativité. À l'inverse, une défaillance de la conduite de groupe laisse la place à des phénomènes régressifs créant de l'insécurité et empêchant tout travail d'élaboration et de transformation collective des informations (Chevetzoff, 2015). Pour soutenir la production du groupe, la conduite de groupe repose sur la fonction de facilitation orientée sur la réalisation de la tâche assignée au groupe et la fonction de régulation visant à assurer que la vie affective du groupe contribue positivement aux objectifs.

Les modalités de conduite du groupe sont donc essentielles pour que le groupe soit le lieu de confrontation des expériences singulières, pour qu'il permette d'élaborer des savoirs partagés, d'accompagner la transformation des identités et d'individualiser les parcours. Les règles d'interaction (confidentialité, discrétion, attention, bienveillance, non-jugement, équité de prise 
de parole et d'écoute) posées par le conducteur de groupe contribuent à un cadre protecteur des interactions.

La méthode d'analyse de pratique, utilisée dans les analyses de situations répond à de telles exigences. La démarche d'analyse de pratique s'est déployée tout d'abord dans les champs du médical et du social pour des professionnels pour lesquels la dimension relationnelle et subjective est essentielle dans leurs pratiques (Lagadec, 2009). Cette approche se diffuse aujourd'hui sous une forme très proche, nommée «co-développement (Champagne et Payette, 2012). L'analyse de la pratique est une forme d'apprentissage qui s'appuie sur l'expérience individuelle et les interactions avec les autres membres du groupe. Un des participants expose une situation problème, puis le groupe par son questionnement et ses associations aide l'exposant à élargir ses représentations de la situation et à explorer sa propre équation personnelle : ressentis, a priori, cadres de référence, éléments de son histoire personnelle et professionnelle qui peuvent introduire un biais dans la perception, compréhension et résolution de la situation. Cette analyse de la situation permet d'élargir le spectre des hypothèses d'interprétations et de solutions. Ce travail de réflexion particulier peut se prolonger par une réflexion plus généralisée et théorique faisant appel aux références conceptuelles des uns et des autres.

Il ne s'agit pas d'une étude de cas, objectivée et à distance de soi. L'objet traité, c'est la relation du professionnel à la situation étudiée. On considère que le sujet, dans une vision systémique, fait partie de la situation problème. Le but de l'analyse de pratique est formatif : il s'agit d'améliorer la conscience de son action dans son environnement professionnel. La logique d'élaboration et d'apprentissage prévaut sur une logique de production d'une solution opérationnelle qui risquerait de renforcer la recherche d'une expertise au détriment d'une élaboration collective.

Les analyses s'élaborent à partir de la subjectivité de la personne qui expose et des représentations et référentiels multiples des membres du groupe. Le groupe et le conducteur sont en position de soutien et d'aide à la réflexion. Au-delà d'une mise en mouvement de la question traitée et de celui qui la porte, la diversité des points de vue des participants constitue une source importante de formation et de questionnement des pratiques de chacun. Questionnements, résonances, analogies, comparaisons et empathie font que l'analyse d'une situation d'un seul profite à tous. 
Ainsi, la dynamique de groupe se construit progressivement, grâce à la confiance, à la sincérité et au partage. La confiance étant construite sur le cadre des règles d'interactions énoncées et tenu par le conducteur; elle contribue à la consolidation du groupe d'appartenance et d'une identité professionnelle collective.

\subsection{Le retour d'expérience comme soutien au processus de construction identitaire}

Une formation diplômante comme la formation d'ingénieur s'accompagne d'un changement de position sociale et d'une transformation de l'identité professionnelle. Jean-Marie Barbier (1996) définit l'identité comme un ensemble de représentations, opératoires (compétences, capacités, habiletés, savoirs formels et savoirs pratiques) et affectives (dispositions génératrices de pratiques, goûts, intérêts, envies) produit par une histoire particulière et dont un individu est le support ou le détenteur à un moment de cette histoire. Les séances consacrées à intégrer l'expérience dans la réflexion sur la dynamique professionnelle font émerger des critères de choix très différenciés d'un étudiant à un autre quant à leurs ambitions professionnelles et familiales, leurs intérêts (chantier, études, recherche, management, etc.), leurs lieux de vie, leurs affinités quant aux structures d'organisation, l'environnement culturel et le rythme de travail.

Le paradoxe est que cette élaboration différenciant les individus est renforcée par la dynamique du groupe et par le sentiment d'appartenance à la communauté des étudiants ingénieurs (Rouchy, 1998). L'étayage par le groupe rend le processus d'individuation plus solide car la dynamique personnelle est autorisée et soutenue par le groupe. L'individu peut s'appuyer sur le socle des valeurs et de représentations communes de son groupe de référence actuel pour affirmer ses différences et son originalité. Mais là encore, ce processus de différenciation n'est possible que si les processus de groupe à l'œuvre sont suffisamment régulés pour éviter des phénomènes régressifs telle que la fusion ou $l^{\prime}$ «illusion groupale » qui vont dénier les différences au lieu de les valoriser (Anzieu, 1999). Pour Didier Anzieu, les situations de groupe ravivent les conflits en rapport à l'identité. Chaque membre du groupe renvoie à chacune des représentations différentes de lui-même. La situation de groupe en formation confronte d'emblée chaque participant à la question : «qui suis-je ?»(Kessar, 2015). Mais si le groupe peut être vécu comme menaçant, il peut apparaître aussi au contraire comme soutien et réparateur. Le processus d'analyse en groupe est parfois un soin à l'identité blessée par le mépris, l'exclusion, l'absence de sens, la frustration ou l'inactivité. Élaborés avec pudeur dans un groupe bienveillant et contenant, des ressentis qui ont été vécus dans l'isolement trouvent un réel soulagement et permettent parfois une restauration narcissique de l'image de soi. Le 
retour d'expérience en groupe accompagne la transformation identitaire du sujet à condition que le dispositif soit suffisamment contenant (Rouchy, 1998). Dans un effet de renforcement positif, cette mise au travail des sujets soutient la rencontre et le travail avec les autres. Pour que les individus puissent connaître l'éprouvé du plaisir partagé à penser et à construire ensemble, l'espace de groupe doit favoriser entre les participants un minimum d'intersubjectivité (Sirota, 2017).

\section{Formulation des questions}

Au regard de ces références conceptuelles, l'enjeu est de penser un dispositif pédagogique qui puisse renforcer la capacité des étudiants à développer des compétences collaboratives à travers la confrontation à la diversité des expériences de leurs pairs, à construire du savoir collectif, à intégrer ces savoirs dans leurs dynamiques professionnelles et à soutenir leur construction identitaire.

Pour mieux comprendre les effets possibles de ce dispositif sur la collaboration, nous tenterons de répondre aux questions suivantes : la mise en place d'espaces groupaux centrés sur la confrontation des différences et des singularités des expériences répond-elle à cet enjeu de développement d'aptitudes au travail collaboratif? L'analyse d'expériences pratiques et singulières en groupe contribue-t-elle à la construction de savoirs psychosociaux ?

\section{Méthodologie et interprétation des données issues des questionnaires d'évaluation du séminaire}

Pour répondre aux questions de la recherche, nous nous sommes appuyé sur l'observation et les évaluations de quatre années du séminaire (2012 à 2015) de «retour d'expérience du stage d'ingénieur », dispositif toujours actif à l'École des Ponts ParisTech. Le séminaire visé, comme tous les modules de l'École des Ponts ParisTech, est en effet évalué par le biais d'un questionnaire à choix multiples associé à des questions ouvertes sur l'ensemble du dispositif et sur chaque séquence. Chaque année, environ la moitié des étudiants ayant participé au séminaire ont rempli le questionnaire (voir le tableau qui suit).

La synthèse de ces questionnaires a été réalisée par un étudiant de chaque groupe. Ces synthèses ont été discutées lors de réunions d'évaluation regroupant les représentants des étudiants, les enseignants et un représentant du service de l'évaluation.

Les données présentées ici sont constituées de : 
- de réponses quantitatives à trois questions (voir tableau ci-dessous) et des commentaires qui leur sont associés ;

- des réponses à une question ouverte « qu'avez-vous appris cette semaine ?».

\begin{tabular}{|l|r|r|r|r|}
\hline & $\mathbf{2 0 1 2}$ & $\mathbf{2 0 1 3}$ & $\mathbf{2 0 1 4}$ & $\mathbf{2 0 1 5}$ \\
\hline Nbre étudiants ingénieurs sollicités & 27 & 96 & 103 & 110 \\
\hline Nbre répondants & 19 & 46 & 58 & 60 \\
\hline \% étrangers & $63 \%$ & $65 \%$ & $72 \%$ & $42 \%$ \\
\hline Pensez-vous que ce module doit être maintenu ? & & & & \\
\hline oui, sans changement & $42 \%$ & $65 \%$ & $43 \%$ & $35 \%$ \\
\hline oui, avec des changements mineurs & $58 \%$ & $26 \%$ & $40 \%$ & $50 \%$ \\
\hline oui, avec des changements majeurs & 0 & $7 \%$ & $15 \%$ & $10 \%$ \\
\hline non & 0 & $2 \%$ & $2 \%$ & $5 \%$ \\
\hline sans changement ou avec changements mineurs & $100 \%$ & $91 \%$ & $83 \%$ & $85 \%$ \\
\hline L'atelier d'analyse de situation était intéressant & & & & NC \\
\hline plutôt oui & $100 \%$ & $93 \%$ & $91 \%$ & \\
\hline plutôt non & 0 & $7 \%$ & $9 \%$ & \\
\hline L'atelier projet professionnel était intéressant & & & & NC \\
\hline plutôt oui & $100 \%$ & $93 \%$ & $90 \%$ & \\
\hline plutôt non & 0 & $7 \%$ & $10 \%$ & \\
\hline
\end{tabular}

Le principal indicateur est l'addition des deux premières réponses à la question «Pensez-vous que ce module doit être maintenu ? Oui, sans changement / oui, avec des changements mineurs / oui, avec des changements majeurs / non ». Cet indicateur atteint $100 \%$ en 2012 (année d'expérimentation avec seulement trois groupes au lieu des huit, les années suivantes), $91 \%$ en 2013, $83 \%$ en 2014, $85 \%$ en 2015. Au regard du taux moyen (86 \%) de ce critère pour les modules de la formation d'ingénieur à l'École des Ponts ParisTech, ces résultats révèlent une satisfaction globale de la part des étudiants. Un commentaire d'un étudiant pourrait résumer les commentaires de satisfaction associés: «Cette semaine est très riche et me semble indispensable. C'est le premier moment que l'on a pour vraiment réfléchir à notre expérience de stage. C'est aussi un bon moyen d'avancer dans notre réflexion et notre projet, tout en tissant des liens avec les étudiants que nous ne connaissons pas. »

\subsection{Appréciations des étudiants sur les différentes séquences du séminaire}


Si les séances d'analyse de situation sont appréciées (100\%, $93 \%, 91 \%)$, c'est, d'après les étudiants, qu'elles «sont les plus interactives» et qu'elles permettent de «partager nos expériences », «d'apprendre de l'expérience des autres »; «relativiser mon expérience », «avoir des outils utiles pour analyser mon expérience », «mieux comprendre les raisons structurelles qui peuvent être derrière les problèmes », «comprendre la complexité du fonctionnement des organisations, analyser l'impact du côté relationnel et managérial sur le déroulement d'une mission »; «acquérir une méthodologie pour analyser et résoudre des problèmes $\gg .$. Les remarques relatives aux 7 ou $9 \%$ d'étudiants insatisfaits de ces ateliers, concernent en particulier «la durée et le côté répétitif de l'analyse de certaines situations », et le fait «que de nombreux étudiants n'ont pas de situation problématique à partager ».

Les séances de travail sur la dynamique professionnelle sont jugées utiles (100 à $90 \%$ des étudiants) car elles permettent «d'actualiser le projet professionnel »; et «... qu'au lieu d'un projet professionnel fermé, j'ai appris à observer les valeurs et les finalités que j'utiliserai pour juger des opportunités auxquelles j'ai accès ».

La composition diversifiée des groupes (en termes d'origine culturelle, de département métier, de sexe, de stage court ou long) a plutôt été appréciée bien que certains eussent préféré « être regroupés par domaines métiers pour la réflexion sur le projet professionnel afin d'ouvrir la connaissance du milieu ».

\section{2. «Qu'avez-vous appris cette semaine?»}

Les 154 réponses apportées à cette question par les 183 répondants ont été classées en plusieurs catégories en fonction de leur contenu (une réponse peut contenir 2 ou 3 thèmes) :

- Échange, partage, écoute et compréhension des autres : 88 occurrences

- Réflexion sur soi, ses valeurs, sa dynamique personnelle : 58 occurrences

- La compréhension du fonctionnement des organisations et du management : 34 occurrences

- La capacité à analyser une situation, à prendre du recul : 34 occurrences

- Un apprentissage de la conduite de groupe : 6 occurrences

- La capitalisation du stage : 7 occurrences

\subsection{L'expérience positive des interactions groupales comme vecteur d'apprentissage des conditions du travail collaboratif}


Ces données de l'évaluation mettent en évidence que l'expérience des interactions positives d'écoute, d'échange et d'apprentissage entre pairs constitue, pour les étudiants, le principal acquis de cette semaine formulé par certains étudiants en termes de «partage des expériences des différentes situations vécues par les autres », «écouter les différentes expériences des autres », ou « avoir énormément appris des autres ».

Les étudiants ont appris à valoriser l'expérience des autres, être à leur écoute, considérer que les expériences des autres étaient source d'apprentissage pour eux-mêmes. Ils ont valorisé les différences, l'écoute de l'autre et l'apprentissage « horizontal » entre pairs.

L'apprentissage se situe autant dans le processus collaboratif que dans les savoirs issus des analyses des expériences. Une remarque sans complaisance d'un des neuf étudiants qui souhaitent la suppression de ce module souligne paradoxalement l'essentiel : « la seule utilité (de ce séminaire) est l'échange d'expérience entre les étudiants... ».

Les étudiants y ont expérimenté les conditions nécessaires à l'apprentissage collaboratif : un cadre et une conduite de groupe qui permettent une circulation confiante et égalitaire de la parole et le dépassement des différences. Trois commentaires d'étudiants : «intervenante qui par ses relances pertinentes et sans jamais couper la parole, a instauré un contexte propice au dialogue ; on se sentait bien écoutés... »; «l'intervenant a su profiter de la diversité de nos parcours pour optimiser notre apprentissage... »; « écouter la situation et le projet de tous les étudiants dans un groupe est nécessaire afin de tous se connaître et d'être sur un même pied d'égalité... ».

L'éprouvé d'une satisfaction d'un échange et d'un partage entre pairs marque la principale acquisition de ce séminaire. Avec 58 occurrences de citation, la réflexion sur soi en situation professionnelle et sur sa dynamique professionnelle constitue l'autre axe fort des acquis : «le principal intérêt était d'identifier mes réactions aux situations de travail et comment je pourrai me comporter face à elles »; «j'ai appris de nouvelles techniques pour faire avancer notre propre réflexion sur notre projet professionnel ». Cette réflexivité s'opère, d'après les verbatim, grâce aux interactions avec les autres au sein des petits groupes : «Découvrir nous-mêmes à travers les autres », « discuter de mon expérience à travers les autres », « mieux comprendre ma première expérience et la comparer avec celles des autres », «écouter les expériences des autres et s'y reconnaître ».

La mise en jeu de la subjectivité de chacun dans les échanges avec les autres à l'occasion d'une analyse réflexive sur son expérience permet la rencontre de sujet à sujet. De cette 
intersubjectivité jaillit une des conditions nécessaires à la collaboration au sein d'un groupe (Sirota, 2017).

Comme nous l'avons développé dans notre cadre conceptuel, la diversité dans la composition des groupes restreints, la conduite de ces groupes par des experts psychosociologues avec des méthodes d'animation participatives et impliquantes comme l'analyse de pratique ont entrainé les étudiants à du co-apprentissage réflexif et leur ont permis d'expérimenter les conditions favorables au travail collaboratif.

\subsection{La construction et la transmission de savoirs issus du partage d'expériences en groupe}

L'acquisition d'une capacité à analyser un contexte socio-organisationnelle dans ce séminaire transparaît à travers les 33 commentaires spontanés des étudiants ingénieurs dont un les résume ainsi : «j'ai appris la complexité des situations professionnelles: comprendre le fonctionnement des organisations, analyser le côté relationnel (par rapport à une personne, groupe ou institution) et managérial qui importe autant que le côté technique, comprendre son impact sur le déroulement d'une mission... ». Les étudiants situent cet apprentissage dans l'analyse des situations en groupe, un commentaire : "les témoignages des autres étudiants permettent de cerner les différentes structures en entreprise que nous n'avons pas pu expérimenter nous-mêmes...».

Concernant les apports théoriques, très peu cités comme valeur ajoutée du séminaire, les constats des étudiants convergent ; des étudiants écrivent : «ce qui m’a le moins intéressé, les exposés des intervenants, l'intérêt d'un tel module résulte de ce qu'apporte chaque étudiant et ce type d'exposé ajoute une dissymétrie malvenue »; «les présentations cassent le rythme des échanges ....»; «j'aurais aimé faire plus d'activités en groupe et moins de conférences...»; «il faut donner plus d'importance aux expériences des étudiants et moins à la partie théorique »... Cette remarque ne révèle pas une dépréciation de la théorie, très valorisée par les ingénieurs, mais résulte de l'expérience éprouvée de la construction collaborative d'un savoir dans une démarche inductive de généralisation d'expériences individuelles.

La plupart des étudiants apprécient la pédagogie de cette approche : « de cette démarche inductive, on a pu relever des enseignements beaucoup plus marquants que la démarche déductive », «les ateliers (thématiques) permettent d'interpréter nos expériences personnelles sous un angle bien précis qui permet de pousser la réflexion assez loin. De plus, cela assure un vrai dynamisme de la part de toutes les personnes impliquées ». 
Toutefois, certains peuvent être gênés par cette inversion de la hiérarchie des savoirs et la démarche inductive, tel cet étudiant : «(dans les ateliers thématiques), où l'on a fait que discuter (en groupes d'étudiants) de ce l'on pensait d'un sujet, en tant qu'étudiant, je ne voyais pas l'intérêt : en général, dans les autres cours, c'est l'inverse, on apprend quelque chose d'un expert ».

Se situant dans le cadre de la formation initiale, l'articulation entre expérience et savoirs formels est une préoccupation majeure dans ce dispositif. Dans un environnement académique comme celui d'une école d'ingénieur, le modèle dominant reste celui de la transmission descendante de savoirs théoriques.

Si les étudiants ont souligné la nécessité d'éviter des ruptures dans la démarche participative par des exposés d'experts au profit des échanges entre pairs et des apports au fil de l'eau des enseignants conducteurs des groupes, c'est qu'ils ont été sensibles au processus à l'œuvre dans ces groupes d'apprentissage en particulier dans la dimension de transmission de savoirs pratiques et savoir-faire.

Les évaluations successives ont conduit à des ajustements du séminaire. Les séances d'analyses de situation et de réflexion sur la dynamique professionnelle ont été très peu modifiées durant ces cinq années. En revanche, les apports théoriques sous la forme d'exposés en amphi ont progressivement disparu. Les apports sont à présent disponibles en ligne ou réalisés « au fil de l'eau » par les enseignants dans chaque atelier.

Les conférences ont été remplacées par un temps d'élaboration entre étudiants : les étudiants identifient et sélectionnent des thèmes issus des analyses de situations qui sont ensuite approfondis en groupe, puis restitués en amphi lors de la dernière séance plénière, sous la forme de scénettes ou de vidéos comme en 2017. Dégagées d'une prétention théorique, ces productions thématiques collectives finales ont la qualité de témoignages concrets et de conseils pratiques. Ces vidéos vont être utilisées pour alimenter un dispositif de préparation au stage, en amont de leurs missions en entreprise. Une véritable boucle de rétroaction positive devrait donc compléter le dispositif de retour d'expérience pour transmettre des savoirs pratiques aux générations suivantes.

L'expérience d'un apprentissage par les pairs à partir de l'expérience terrain contribue à donner une place aux savoirs issus de l'expérience à côté des savoirs académiques. 


\subsection{Le retour d'expérience par des analyses de situation en groupe comme contribution à l'acquisition de compétences psychosociales}

La réflexivité sur l'expérience professionnelle et l'expérience du groupe comme support d'un apprentissage ont permis de se décentrer du modèle de transmission descendante de savoirs théoriques par des experts pour s'ouvrir à un modèle d'acquisition de savoirs pratiques élaborés entre pairs dans des liens intersubjectifs.

Les activités réalisées en groupe par les étudiants ont mobilisé leur écoute et leurs capacités à se décentrer, à mettre en jeu leur subjectivité dans les échanges avec les autres, à reconnaître dans les autres une identité différente de la leur et à s'en enrichir. L'exercice de cette posture de disponibilité et d'accueil des autres dans un groupe constitue pour nous une compétence psychosociale fondamentale pour toutes les interactions de collaboration.

Une autre compétence psychosociale mobilisée dans ce dispositif est la capacité à analyser et apprendre de son expérience par l'analyse socio-organisationnelle de situations vécues dans un contexte professionnel. Cette compétence réflexive contribue à la construction identitaire des individus. Elle a été mobilisée en particulier dans les questionnements sur le projet professionnel.

\section{En conclusion}

À partir de l'analyse du dispositif de retour d'expérience du stage des étudiants ingénieurs de l'école des Ponts ParisTech au regard des théories du groupe, l'expérience de l'apprentissage par le groupe apparaît comme favorisant l'acquisition de compétences psychosociales, en particulier d'aptitudes au travail collaboratif en milieu complexe.

Le retour d'expérience du stage d'ingénieur est le lieu pour nouer des liens entre les singularités des apprenants et la diversité des expériences vécues. Ce retour d'expérience s'appuyant sur des groupes d'analyse restreints apparaît comme un outil de cohésion réinsérant les étudiants dans le dispositif de formation après un an d'interruption et établissant un lien entre les apprentissages issus de l'expérience et les savoirs académiques de la troisième année. Il affirme aussi la différence et la diversité soutenant l'individualisation des parcours.

Les étudiants repartent avec une nouvelle expérience de travail de groupe et d'apprentissage par les pairs, une nouvelle méthode de conduite de groupe, et de nouvelles connaissances quant au fonctionnement des individus au sein d'équipes dans une institution. L'approche 
pédagogique analysée aide les étudiants à passer du «penser à », solitaire, au penser «avec » d'autres sujets pensants.

La nature des échanges, les grilles d'interprétation issues de la psychosociologie et l'expérience de groupe contribuent au développement de compétences psychosociales telles que l'écoute, la capacité à apprendre des autres, à analyser en groupe, à renforcer son « je » professionnel par la réflexivité tout en contribuant à soutenir celles des autres, toutes compétences qui devraient faire partie du référentiel de compétences de l'ingénieur.

Pour mesurer les effets de ces acquisitions, il serait intéressant, dans un moyen terme, de voir de quelle manière elles ont un impact sur le niveau de collaboration et la qualité des échanges des travaux de groupe de troisième année et sur le stage de fin d'étude ; et à long terme, de vérifier l'exercice de ces compétences dans le cadre de leur premier emploi.

Cette étude ouvre sur des perspectives d'approfondissement concernant les effets d'un travail réflexif en groupe sur la transformation identitaire des étudiants ingénieurs. En effet, au-delà de la fonction de soutien face à des vécus parfois difficiles et d'étayage de la singularité de chacun par les échanges, on peut faire l'hypothèse que l'élaboration en groupe issue des vécus individuels contribue à la construction identitaire et professionnelle des étudiants.

Le groupe constitue un puissant levier de transformation du rapport au savoir indispensable pour préparer les étudiants à la complexité du monde professionnel, exigeant de plus en plus un travail et un apprentissage collaboratif pour faire face à l'accélération des évolutions sociotechniques.

\section{Références bibliographiques}

Agence Nationale d'amélioration des conditions de travail. (2017). Apprendre à manager le travail. Livre blanc des initiatives en formation initiale et continue. Lyon : Anact.

Anzieu, D. (1999). Le groupe et l'inconscient. Paris : Dunod.

Argyris, C. et Schön, D. A. (1996, 1978). Apprentissage organisationnel - Théorie, méthode, pratique. Bruxelles : De Boeck Université.

Barbier, J.-M. (1996). Analyse des pratiques : questions conceptuelles. Dans C. BlanchardLaville, et D. Fablet (dir.). L'analyse des pratiques professionnelles (p. 27-49). Paris : L'Harmattan.

Champagne, C et Payette, A. (2012). Le groupe de codéveloppement professionnel. Québec: Presses de l'Université du Québec. 
Chevetzoff, P. (2015). Méthodologie de conduite de groupe dans la perspective de l'ARIP/Transition. Connexions, 104, 19-34.

De Gaulejac, V. (1992). La névrose de classe. Paris : Hommes et groupes.

Kessar, Z. (2015). Conduite de groupe et intervention. Connexions, 104,103-117.

Lagadec, A.-M. (2009). L'analyse des pratiques professionnelles comme moyen de développement des compétences : ancrage théorique, processus à l'œuvre et limites de ces dispositifs. Recherche en soins infirmiers, 2(97), 4-22.

Le Roy, J. (2017). L'inter/transculturel dans la pensée de Jean-Claude Rouchy. Connexions, 108, 83-90.

Meirieu, P. (1997). Groupes et apprentissages. Connexions, 69, 13-29.

Rouchy, J.-C. (1998). Le groupe, espace analytique. Toulouse : Érès.

Rouchy, J.-C. et Soula-Desroche, M. (2004). Institution et changement. Toulouse : Érès.

Sirota, A. (2017). Pervers narcissiques : comprendre, déjouer, surmonter. Paris : Le Manuscrit.

Schön D. (1994). Le praticien réflexif, à la recherche du savoir caché dans l'agir professionnel. Montréal : Les Éditions Logiques. 